UCRL-CR-125214

S/C- B299142

\title{
Reconnection Methods for an Arbitrary \\ Polyhedral Computational Grid
}

V. V. Rasskazova, I. D. Sofronov, A. N. Shaporenko,

D. E. Burton, D. S. Miller

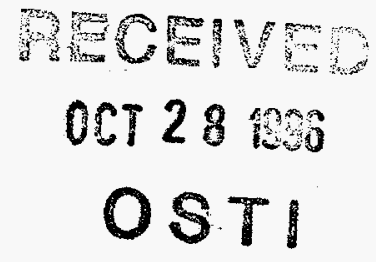

August 22, 1996

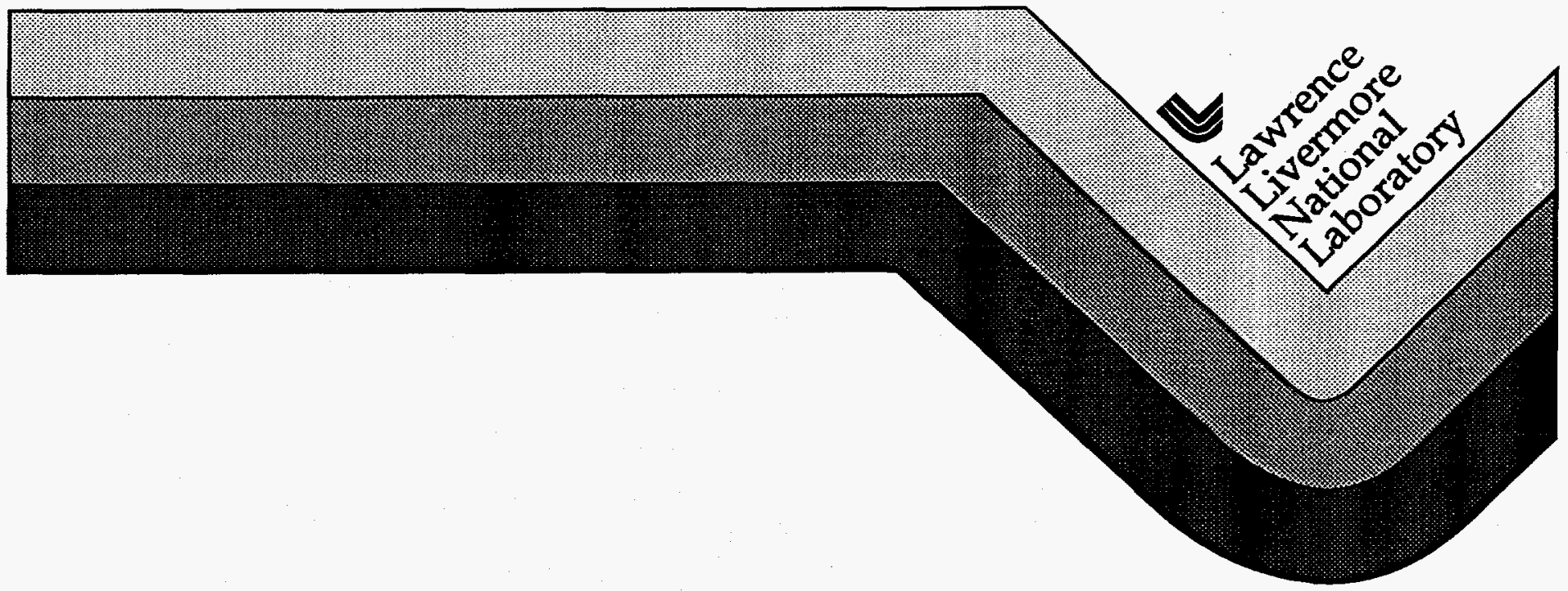

LL6421* v1.0 (3/96) 


\section{DISCLAIMER}

Portions of this document may be illegible in electronic image products. Images are produced from the best available original document. 


\title{
Russian Federal Nuclear Center
}

Arsamas-16, Nizhni Novgorod Region, Russia, 607200

\section{RECONNECTION METHODS FOR AN ARBITRARY \\ POLYHEDRAL COMPUTATIONAL GRID}

\begin{abstract}
Report
under U.S. University California( Lawrence Livermore National Laboratory) -.. Russian Federal Nuclear Center (VNIIEF) Contract No. B299142.
\end{abstract}

V.V.Rasskazova, I.D.Sofronov, A.N.Shaporenko

University Technical Representatives

Dr. D.E.Burton

from LLNL

Dr. D.S.Miller

Principal Investigator from VNIIEF

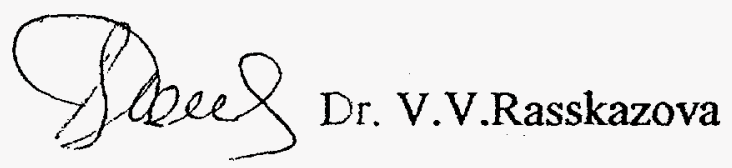




\begin{abstract}
The paper suggests a method for local reconstructions of a 3D irregular computational grid and the algorithm of its program implementation. Two grid reconstruction operations are used as basic: paste of two cells having a common face and cut of a certain cell into two by a given plane.

This paper presents criteria to use one or another operation, the criteria are analyzed.

A program for local reconstruction of a 3D irregular grid is used to conduct two test computations and the computed results are given.
\end{abstract}

\title{
INTRODUCTION
}

The Lagrangian method is as extremely powerful technique for solving gas dynamics problems. However, its drawback is that when flows with severe material strains are computed grid distortion takes place which, in its turn, leads to decreasing timestep value and in some cases to impossibility of further proceeding with the computations without preliminary grid reconstruction.

Lagrangian grid reconstruction was in common practice mainly in 2D codes and, besides, as applied to regular (quadrangular) grids.

Recently interest has quickened in various nonstructured and irregular grids which are much more amenable to various kinds of reconstruction and, moreover, allow to localize the grid segments subject to reconstruction.

Thus the problem arose to develop a technique and program allowing to maintain an acceptable grid during the whole computation of a gas-dynamical problem on the Lagrangian irregular grid whose cells in two dimensions are polygons. Operation of the developed program showed that it can be used to solve practically any gas-dynamical problems, including problems with severe material strains and vorticity flows. At local reconstructions all problem integral characteristics are retained which positively tells on the computation accuracy.

Taking into consideration all positive qualities of the program for local reconstructions of the 2D irregular Lagrangian grid, as well as gained experience we made an attempt to develop the technique and program for reconstruction of $3 \mathrm{D}$ grids composed of arbitrary polyhedra. 
Thus, this paper is devoted to consideration of issues of development of the technique for local reconstructions of $3 \mathrm{D}$ grids whose cells are represented with Voronoi bodies $/ 1,2,3 /$ at the initial time and with arbitrary polyhedra during further grid deformation and reconstruction. A particular attention is therewith paid to the aspects of grid geometry reconstruction and architectural connections. The paper describes the criteria for local grid reconstructions, as well as results of two model computations are given.

\section{LOCAL RECONSTRUCTIONS OF 3D IRREGULAR LAGRANGIAN COMPUTATIONAL GRID}

Preparatory to direct description of the local reconstruction method itself, it is necessary to introduce the notion of the topologic grid structure this method is designed for.

As it was mentioned, in two dimensions irregular grid cells are represented with arbitrary polygons whose vertices are grid nodes $/ 4 /$. The topologic structure of the grid is determined with connections of its nodes. Each 2D grid node has exactly three neighbors. Similarly, in three dimensions cells are represented in the general case with arbitrary polyhedra and the number of connections in each node equals four. This grid feature was determining at development of the method of local grid reconstructions.

The local grid reconstruction operations in two dimensions were based on two basic operations: paste of two cells having a common edge and cut of one cell into two with some straight line. Introduction of just these two operations is accounted for by the following considerations:

1) both the operations are correspondent with the basic requirement of grid reconstruction, that is allow to refine or coarsen the grid, if needed;

2) the cell cut and paste operations do not alter the topological structure of the grid;

3) being elementary operations on grid cells, these operations can be used as blocks to construct other, more complex grid reconstruction operations.

Basing on the experience of using the grid cell cut and paste operations in two dimensions try to apply these operations to 3D polyhedral grid reconstruction.

By analogy with two dimensions we base on the fact that the volume of the cells surrounding those being reconstructed does not vary at cell cut and paste operation execution. This condition is useful both at 
grid gas-dynamical value updating and when the condition of convexity of all the cells upon grid reconstruction is met.

To meet the condition laid down, find the dependence of cell volume variation on displacement of one of the cell nodes.

\subsection{Variation in cell volume at displacement of an arbitrary cell node.}

Consider an arbitrary polyhedral cell (Fig.1).

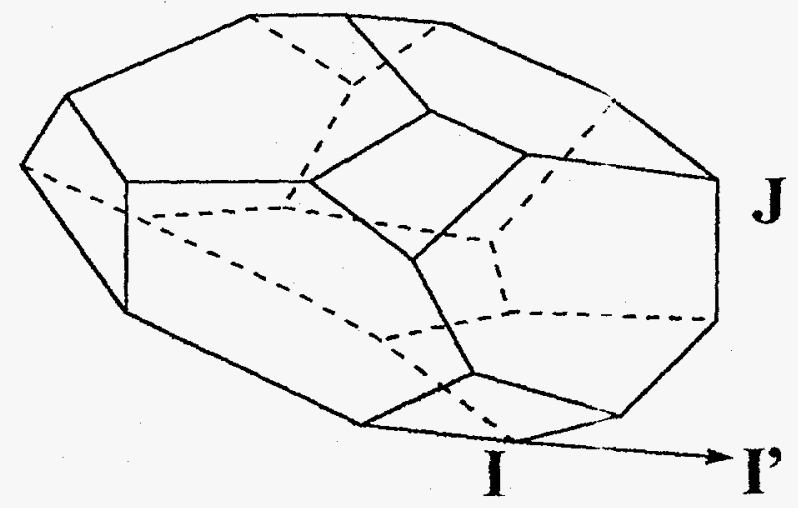

An irregular 3D grid cell.

Fig.1.

Suppose that its node I has moved to the point I'. Estimate the variation in the $\mathbf{J}$ cell volume. We compute the cell volume as the sum of volumes of the tetrahedra for which the vertices of each are the center of the cell, the center of the face and two noles of this face. Note that the change in the cell volume will result from deformation of only those tetrahedra which rest on the faces adjacent to the node I. Consider how the volumes of the terahedra adjacent to one of such faces $\mathbf{j}$ will change (Fig.2). 


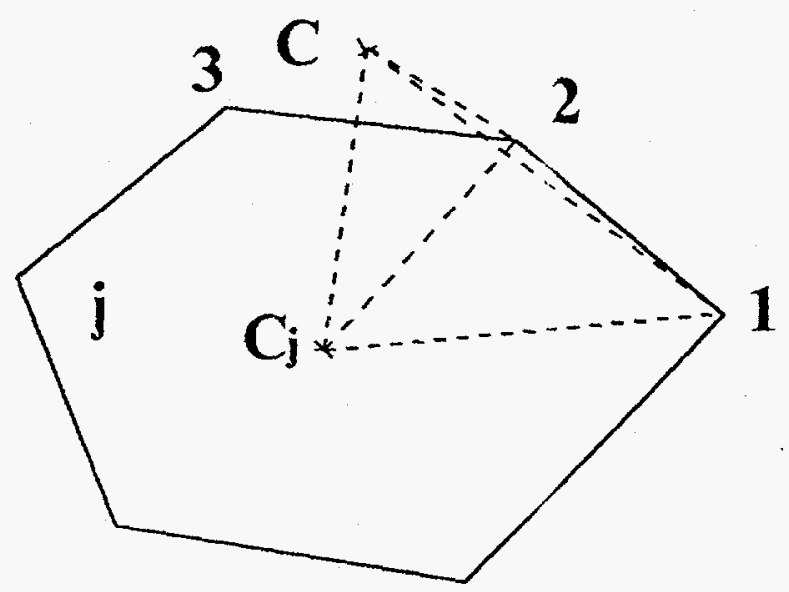

nj

Polyhedral 3D grid cell face

Fig.2.

At an arbitrary displacement of node 1 of the face $\mathbf{j}$ the total change in the volumes of the tetrahedra adjacent to this face is estimated by the relation

$$
\begin{aligned}
& \Delta V_{j}=\frac{1}{6}\left\{\left(\vec{r}_{c}^{j^{\prime}},\left[\vec{r}_{1}{ }^{\prime} \times \vec{r}_{2}^{j}\right]\right)+\left(\vec{r}_{c}^{j^{\prime}},\left[\vec{r}_{n_{j}}^{j} \times \vec{r}_{1}^{\prime}\right]\right)+\right. \\
& \left.\sum_{i=2}^{n_{j}-1}\left(\vec{r}_{c}^{j^{\prime}},\left[\vec{r}_{i}^{j} \times \vec{r}_{i+1}^{j}\right]\right)-\sum_{i=1}^{n_{j}}\left(\vec{r}_{c}^{j},\left[\vec{r}_{i}^{j} \times \vec{r}_{i+1}^{j}\right]\right)\right\},
\end{aligned}
$$

where $\vec{r}_{i}^{j}$ - the radius vector passed from the cell center to the node $i$ of the face $j\left(i=2, \ldots, n_{j}\right)$;

$\rightarrow$

$r_{1}$ - the radius vector passed from the cell center to the node being displaced;

$\vec{r}_{c}^{j}$ - the radius vector passed from the cell center to the $j$ face center;

$\mathrm{n}_{j}$ - the number of nodes of the face $j$ selected counter-clockwise if one looks from the cell center. The dashed values characterize the face after displacement of node 1 .

Summing the volume changes over all faces adjacent to node 1 and collecting similar terms we obtain 


$$
\begin{aligned}
& \Delta V=\frac{1}{6} \sum_{j=1}^{3}\left\{\left({\overrightarrow{r_{2}}}^{j}-{\overrightarrow{r_{n}}}_{j}^{j},\left[{\overrightarrow{r_{c}}}^{j} \times \vec{r}_{1}\right]-\left[{\overrightarrow{r_{c}}}^{j} \times \vec{r}_{1}\right]\right)+\right. \\
& \left.\sum_{i=2}^{n_{j}-1}\left({\overrightarrow{r_{c}}}^{j}-{\overrightarrow{r_{c}}}^{j},\left[{\overrightarrow{r_{i}}}^{j} \times{\overrightarrow{r_{i+1}}}^{j}\right]\right)\right\} .
\end{aligned}
$$

Since in the expression for $\vec{r}_{c}^{j^{\prime}}$ the coordinates of the vector $\overrightarrow{r_{1}^{\prime}}$, appear then the dependence of volume change on the coordinates of node 1 proves to be quadratic.

However, if ${\overrightarrow{r_{c}}}^{j^{\prime}}$ is taken to be arithmetical mean of vectors $\vec{r}_{i}^{j}$ then $\vec{r}_{c}^{j^{\prime}}=\frac{\overrightarrow{r_{1}^{\prime}}-\overrightarrow{r_{1}}}{n_{j}}+\vec{r}_{c}^{j}$ and the dependency $\Delta \mathrm{V}\left(\overrightarrow{r_{l}^{\prime}}\right)$ becomes linear. On simple transformations we find

$$
\begin{aligned}
& \Delta V=\frac{1}{6}\left(\vec{r}_{1}-\overrightarrow{r_{1}}, \vec{A}\right), \text { where } \\
& \vec{A}=\sum_{j=1}^{3} \frac{1}{n_{j}}\left\{\left[\left(\vec{r}_{n_{j}}^{j}-\vec{r}^{j}\right) \times \sum_{i=2}^{n_{j}^{j}} \vec{r}_{i}^{j}\right]+\right. \\
& \left.\sum_{i=2}^{n_{j}-1}\left[\vec{r}_{i}^{j} \times{\overrightarrow{r_{i+1}^{j}}}^{j}\right]\right\} .
\end{aligned}
$$

This formula is the milestone of the whole method of polyhedral grid local reconstructions. In particular, the corollary of the law derived by us is the fact that when node 1 of cell $J$ moves in the plane given by the equation

$$
\left(x-x_{1}\right) C_{y z}+\left(y-y_{1}\right) C_{z x}+\left(z-z_{1}\right) C_{x y}=0 \text {, }
$$

where $C_{x y}=\left(\vec{e}_{z}, \vec{A}\right), C_{y z}=\left(\vec{e}_{x}, \vec{A}\right), C_{z x}=\left(\vec{e}_{y}, \vec{A}\right)$,

and $\overrightarrow{e_{x}}, \overrightarrow{e_{y}}$ and $\overrightarrow{e_{z}}$ - unit vectors of coordinate axes the cell volume does not change.

\subsection{Cell paste}

Consider two arbitrary cells, $J_{I}$ and $J_{2}$, having a common face (Fig.3). 


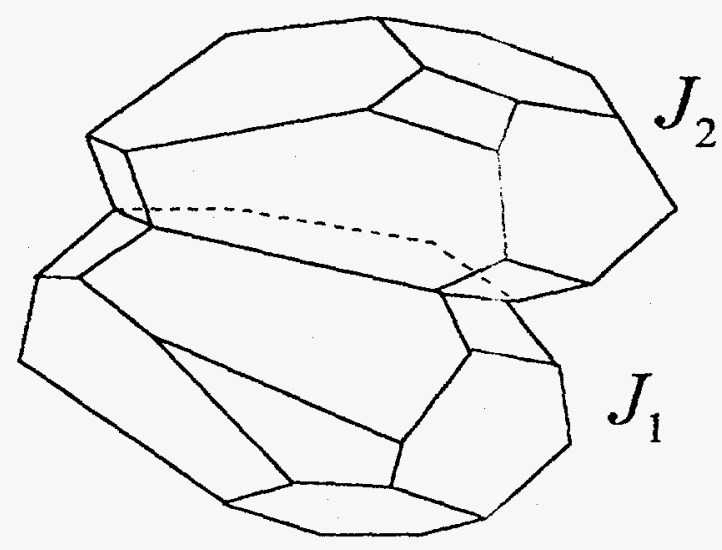

Two cells with a common face.

Fig 3.

Their paste should reduce to removal of the common face, i.e. actual removal of nodes lying on it, from the system of connections. However, in doing so the volumes of the cells adjacent to the cells being pasted will change, hence, the condition of conservation of volumes set up at the beginning of this section will be not met.

To avoid this, it is necessary to move the nodes connected to the common face nodes so that the volumes adjacent to those being pasted be recovered.

Let $\Delta V_{k j}$ be the changes in the volums of the cells having common faces simultaneously with cells $\mathbf{J}_{1}$ and $\mathbf{J}_{2}$. Consider two such neighboring cells $\mathbf{K}_{1}$ and $\mathbf{K}_{2}$ (Fig.4).

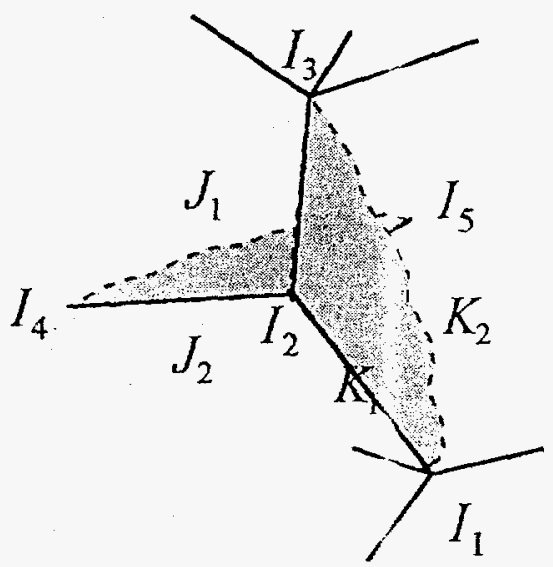

3D irregular grid element at junction of four cells.

Fig 4. 
The node $I_{2}$ surrounded by the cells $J_{1}, J_{2}, K_{1}$ and $K_{2}$ is removed from the face common for the cells $J_{I}$ and $J_{2}$, i.e. its connection with the nodes $I_{1}$ and $I_{3}$ is replaced with the connection between the nodes $I_{I}$ and Is.

In order to find new coordinates of the nodes $I_{1}$ and $I_{3}$ for which the volumes of the cells $K_{1}$ and $K_{2}$ do not change, the following algorithm is suggested:

1) Formula (1) is used to compute changes in volumes $\Delta \widetilde{V}_{k 1}$, $\Delta \widetilde{V}_{k 2}, \Delta \widetilde{V}_{j 1}$ and $\Delta \widetilde{V}_{j 2}$ of cells $K_{1}, K_{2}, J_{l}$, and $J_{2}$, at movement of the node $I_{2}$ along the bisector of the angle $I_{I} I_{2} I_{3}$ until intersection with straight line segment $I_{I} I_{3}$. Call these values as test volume variations.

2) As each cell $K_{i}$ possesses two nodes of the face being removed then it is correspondent with two test volume variations: $\Delta \widetilde{V}_{k i}$ and $\Delta \widetilde{\widetilde{V}}_{k i}$. Hence, knowing the true variation in the cell volume $\Delta V_{k i}$ which takes place at removal of the common face of the cells $J_{1}$ and $J_{2}$ one can compute the fraction of variation in the volume $\Delta \bar{V}_{k i}$ for the cell $K_{i}$ due to displacement of the nodes $I_{1}$ and $I_{3}$,

$$
\Delta \bar{V}_{k i}=\Delta \widetilde{V}_{k i} \cdot \frac{\Delta V_{k i}}{\Delta \widetilde{V}_{k i}+\Delta \widetilde{V}_{k i}}
$$

3) Using test volume variations of cells $\Delta \widetilde{V}_{j 1}$ and $\Delta \widetilde{V}_{j 2}$ one can estimate the volume variation fraction corresponding specifically to either of the two nodes $I_{\boldsymbol{I}}$ and $\boldsymbol{I}_{3}$

$$
\begin{aligned}
& \Delta V_{1, k i}=\Delta \widetilde{V}_{j 1} \cdot \frac{\Delta \bar{V}_{k i}}{\Delta \widetilde{V}_{j 1}+\Delta \widetilde{V}_{j 2}} \\
& \Delta V_{3, k i}=\Delta \widetilde{V}_{j 2} \cdot \frac{\Delta \bar{V}_{k i}}{\Delta \widetilde{V}_{j 1}+\Delta \widetilde{V}_{j 2}}
\end{aligned}
$$

4) Now the new position of the node $I_{I}$ is estimated by the equation system: 


$$
\left\{\begin{array}{l}
\left(x_{1}^{\prime}-x_{1}\right) C_{y z}^{1}+\left(y_{1}^{\prime}-y_{1}\right) C_{z x}^{1}+\left(z_{1}^{\prime}-z_{1}\right) C_{x y}^{1}=0 \\
\left(x_{1}^{\prime}-x_{1}\right) C_{y z}^{2}+\left(y_{1}^{\prime}-y_{1}\right) C_{z x}^{2}+\left(z_{1}^{\prime}-z_{1}\right) C_{x y}^{2}=\Delta V_{1, k 1} \\
\left(x_{1}^{\prime}-x_{1}\right) C_{y z}^{3}+\left(y_{1}^{\prime}-y_{1}\right) C_{z x}^{3}+\left(z_{1}^{\prime}-z_{1}\right) C_{x y}^{3}=\Delta V_{1, k 2}
\end{array}\right.
$$

The first equation of this system corresponds to conservation of volume of the $I_{\boldsymbol{I}}$ node cell which does not contain the node $\boldsymbol{I}_{\mathbf{2}}$ and two second equations correspond to recovery of the $K_{1}$ and $\boldsymbol{K}_{2}$ cell volumes. This equation system has a unique solution if its principal determinant is non-zero, i.e.

$$
\left|\begin{array}{ccc}
C_{y z}^{1} & C_{z x}^{1} & C_{x y}^{1} \\
C_{y z}^{2} & C_{z x}^{2} & C_{x y}^{2} \\
C_{y z}^{3} & C_{z x}^{3} & C_{x y}^{3}
\end{array}\right| \neq 0
$$

Geometrically this means that no two planes of the three are parallel to one another. Assume that in this equation system two parallel planes are found. This means that when the node $I_{l}$ with the coordinates $\left(x_{1}, y_{1}, z_{1}\right)$ moves in the plane $\alpha$ retaining the volume of some cell $L_{1}$ the volume of another cell $L_{2}$ is simultaneously retained. As the plane retaining the volume of the cell $L_{l}$ has only one common point with this cell then, hence, it does not also intersect the cell $L_{2}$, i.e. all four nodes connected to $I_{I}$ lie on one side of some plane (Fig.5).

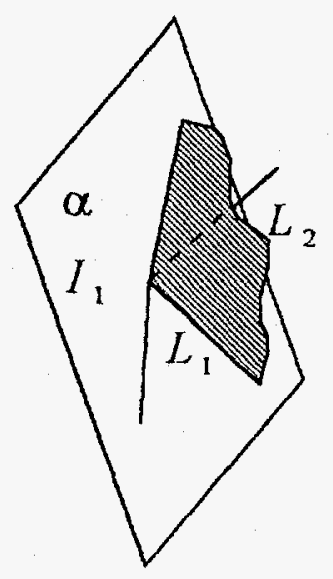

Four connections of node $I_{1}$ lie on one side of plane $\alpha$ Fig 5. 
Therefore, two other cells the node $l_{1}$ belongs to must be concave. Hence, when the determinant of the equation system for estimation of some node displacement equals 0 it is necessary first to correct concavity at that node. The correction of concave cells will be described below. Hence, the geometric aspects of the problem of pasting two cells with conservation of volumes of the cells contacting them are solved.

\subsection{Cell cut}

At cutting a cell $J$ the section plane used to cut the cell into two new should be determined. When all points of intersection of this plane with the cell edges are found and new cell and node connections are determined we obtain two new cells $J_{1}$ and $J_{2}$ (Fig.6).

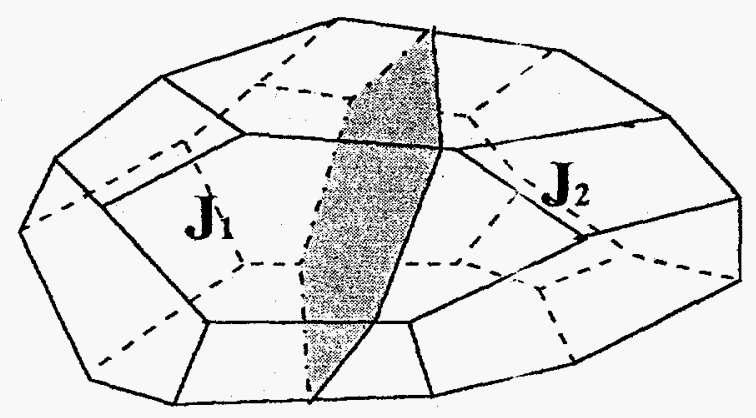

Example of polyhedral cell cut with a plane Fig 6.

However, it should be noted that the volumes of the cells adjacent to the $J$ cell faces intersected by the cut plane may be changed if the above faces are not planar. Adhering to the idea of conservation of cell volumes at execution of the local grid reconstruction operations we should move the cell nodes connected to the nodes of the face appearing at the cut so as to recover the cell volumes, that is make the operation inverse to that which took place at cell paste. By virtue of complete identity of these operations, we will not repeat description of the algorithm. 


\subsection{Correction of concavities}

During local reconstruction concavities may appear which should be corrected to proceed with the computation. Otherwise cells will turn inside out and the grid will become "tangled". We will consider the cell $\boldsymbol{J}$ as concave at the node $I$ if the mixed product of the regular three of vectors is $\left(\vec{r}_{1}, \vec{r}_{2}, \vec{r}_{3}\right)<0$, where $\vec{r}_{k}$ - the vector connecting the node $I$ with its neighbor $I_{k}$, with $k$ being not equal to the address of the cell $J$ in the package of the $I$ node cell connections (Fig.7). Hereinafter for the concavity value we take the absolute value of the mixed product of vectors $\left(\vec{r}_{1}, \vec{r}_{2}, \vec{r}_{3}\right)$.

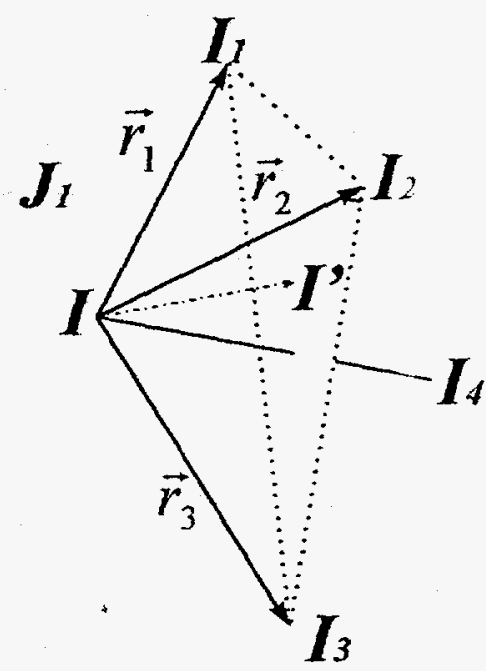

Fragment of polyhedral cell $\boldsymbol{J}$ containing concavity at the node $I$

Fig 7.

The order of the vectors $\vec{r}_{1}, \vec{r}_{2}$ and $\vec{r}_{3}$ in the mixed product is secured by the number of addresses of corresponding nodes in the package of $I$ node connections. The package of node connections is constructed basing on the following principles:

1) Each node is correspondent with four cells surrounding it and four nodes connected to it which can be represented in the form of the following table:

\begin{tabular}{|l|l|l|l|l|}
\hline$I$ & $J_{1}$ & $J_{2}$ & $J_{3}$ & $J_{4}$ \\
\cline { 2 - 5 } & $I_{1}$ & $I_{2}$ & $I_{3}$ & $I_{4}$ \\
\hline
\end{tabular}


2) The node $I_{i}$ located at the $i$-th address of the package $(i=1,2,3,4)$ does not belong to the cell $J$;

3 ) If the node $I$ belongs to the boundary then the lower-numbered addresses contain the numbers of the celis surrounding it and then the numbers of the boundary conditions follow;

4) The cells surrounding the node $I$ will be convex at that node when the following conditions are satisfied:

$$
\begin{aligned}
& \left(\vec{r}_{I I_{1}}, \vec{r}_{I I_{2}}, \vec{r}_{I I_{3}}\right)>0,\left(\vec{r}_{I I_{2}}, \vec{r}_{I I_{4}}, \vec{r}_{I I_{3}}\right)>0, \\
& \left(\vec{r}_{I I_{1}}, \vec{r}_{I I_{3}}, \vec{r}_{I I_{4}}\right)>0,\left(\vec{r}_{I I_{1}}, \vec{r}_{I I_{4}}, \vec{r}_{I I_{2}}\right)>0 .
\end{aligned}
$$

To correct concavity in the cell $J$, move the node $I$ to the point $I$ ' lying on the plane of nodes $I_{1}, I_{2}$ and $I_{3}$ so that variations in the volumes of the other cells of the node $I$ be equal between themselves. This can be easily done using formulas (1), (2).

Evidently, concavity in the node $I$ will be thereby corrected, but, following the idea of conservation of cell volumes at local grid reconstruction, it is necessary to recover the changed cell volumes.

Thus, the problem arises: how one can recover the volumes so that the concavities which can arise in doing so be minimal.

Recover the changed cell volumes through movement of the nodes $I_{1}, I_{2}$ and $I_{3}$. The variations in the volumes of the cells $J_{1}, J_{2}$ and $J_{3}$ at displacement of the node $I$ (consider them as different to retain generality of the conclusions) may be computed by formulas (1), (2). Denote them as $\Delta \mathrm{V}_{1}, \Delta \mathrm{V}_{2}, \Delta \mathrm{V}_{3}$, respectively. By moving the node $I_{1}$ one can simultaneously recover some fractions of volumes of the cells $J_{3}$ and $J_{2}$. Denote the factors of these fractions as $\alpha_{1}$ and $\beta_{1}$, respectively. Then, according to formulas (1), (2), displacement of the node $I_{I}$ is estimated by the linear equation system

$$
\left\{\begin{array}{l}
\left(x_{1}^{\prime}-x_{1}\right) C_{y z}^{1}+\left(y_{1}^{\prime}-y_{1}\right) C_{z x}^{1}+\left(z_{1}^{\prime}-z_{1}\right) C_{x y}^{1}=0 \\
\left(x_{1}^{\prime}-x_{1}\right) C_{y z}^{2}+\left(y_{1}^{\prime}-y_{1}\right) C_{z x}^{2}+\left(z_{1}^{\prime}-z_{1}\right) C_{x y}^{2}=\alpha_{1} \Delta V_{3} \\
\left(x_{1}^{\prime}-x_{1}\right) C_{y z}^{3}+\left(y_{1}^{\prime}-y_{1}\right) C_{z x}^{3}+\left(z_{1}^{\prime}-z_{1}\right) C_{x y}^{3}=\beta_{1} \Delta V_{2}
\end{array}\right.
$$

where coefficients $\mathrm{C}_{x y}, \mathrm{C}_{y z}$ and $\mathrm{C}_{z x}$ are estimated by formulas (2) ,(3). Similar equation systems can be written for the nodes $I_{2}$ and $I_{3}$. The unknown coefficients $\alpha_{i}$ and $\beta_{i}$ can be used for solving the aboveformulated problem of concavity minimization.

Displacement of the node $I_{1}$ can lead to change in volumes of sixteen tetrahedra. Vertices of four of these tetrahedra lie immediately at the point $I_{1}$. Vertices of other twelve tetrahedra lie in the nodes connected to the node $I_{1}$, three in each. Denote the volumes of each 
tetrahedra as $V_{i}(\mathrm{i}=1, \ldots, 16)$ before the displacement of the node $I_{1}$ and as $V_{i}$ after the displacement. Express the valucs $V_{i}^{\prime}$ through $V_{i}, \alpha_{1}$ and $\beta_{1}$.

For tetrahedra immediately connected with the node $I_{I}$ we have in the coordinate form:

$$
\begin{aligned}
& V^{\prime}=\left|\begin{array}{ccc}
x_{1}-x^{\prime} & y_{1}-y^{\prime} & z_{1}-z^{\prime} \\
x_{2}-x^{\prime} & y_{2}-y^{\prime} & z_{2}-z^{\prime} \\
x_{3}-x^{\prime} & y_{3}-y^{\prime} & z_{3}-z^{\prime}
\end{array}\right|=\left|\begin{array}{ccc}
x_{1}-x & y_{1}-y & z_{1}-z \\
x_{2}-x & y_{2}-y & z_{2}-z \\
x_{3}-x & y_{3}-y & z_{3}-z
\end{array}\right|- \\
& -\Delta x \cdot\left|\begin{array}{lll}
1 & y_{1} & z_{1} \\
1 & y_{2} & z_{2} \\
1 & y_{3} & z_{3}
\end{array}\right|-\Delta y \cdot\left|\begin{array}{lll}
x_{1} & 1 & z_{1} \\
x_{2} & 1 & z_{2} \\
x_{3} & 1 & z_{3}
\end{array}\right|-\Delta z \cdot\left|\begin{array}{lll}
x_{1} & y_{1} & 1 \\
x_{2} & y_{2} & 1 \\
x_{3} & y_{3} & 1
\end{array}\right|
\end{aligned}
$$

where $\Delta \mathrm{x}=\mathrm{x}^{\prime}-\mathrm{x}, \Delta \mathrm{y}=\mathrm{y}^{\prime}-\mathrm{y}$ and $\Delta \mathrm{z}=\mathrm{z}^{\prime}-\mathrm{z}$. Having found the values $\Delta \mathrm{x}$, $\Delta y$ and $\Delta z$ from system (4) and taking into consideration that

$$
\begin{aligned}
& V=\left|\begin{array}{lll}
x_{1}-x & y_{1}-y & z_{1}-z \\
x_{2}-x & y_{2}-y & z_{2}-z \\
x_{3}-x & y_{3}-y & z_{3}-z
\end{array}\right| \text { we obtain } \\
& V^{\prime}=V+\frac{1}{d} \alpha \Delta V_{3}\left\{\left|\begin{array}{ll}
C_{z x}^{1} & C_{x y}^{1} \\
C_{z x}^{3} & C_{x y}^{3}
\end{array}\right| \cdot\left|\begin{array}{ccc}
1 & y_{1} & z_{1} \\
1 & y_{2} & z_{2} \\
1 & y_{3} & z_{3}
\end{array}\right|+\left|\begin{array}{ll}
C_{x y}^{1} & C_{y z}^{1} \\
C_{x y}^{3} & C_{y z}^{3}
\end{array}\right| \cdot\left|\begin{array}{lll}
x_{1} & 1 & z_{1} \\
x_{2} & 1 & z_{2} \\
x_{3} & 1 & z_{3}
\end{array}\right|+\right. \\
& \left.+\left|\begin{array}{ll}
C_{y z}^{1} & C_{z x}^{1} \\
C_{y z}^{3} & C_{z x}^{3}
\end{array}\right| \cdot\left|\begin{array}{lll}
x_{1} & y_{1} & 1 \\
x_{2} & y_{2} & 1 \\
x_{3} & y_{3} & 1
\end{array}\right|\right\}-\frac{1}{d} \beta \Delta V_{2}\left\{\left|\begin{array}{ll}
C_{z x}^{1} & C_{x y}^{1} \\
C_{z x}^{2} & C_{y y}^{2}
\end{array}\right| \cdot\left|\begin{array}{lll}
1 & y_{1} & z_{1} \\
1 & y_{2} & z_{2} \\
1 & y_{3} & z_{3}
\end{array}\right|+\right. \\
& \left.+\left|\begin{array}{lll}
C_{x y}^{1} & C_{y z}^{1} \\
C_{x y}^{2} & C_{y z}^{2}
\end{array}\right| \cdot\left|\begin{array}{lll}
x_{1} & 1 & z_{1} \\
x_{2} & 1 & z_{2} \\
x_{3} & 1 & z_{3}
\end{array}\right|+\left|\begin{array}{ll}
C_{y z}^{1} & C_{z x}^{1} \\
C_{y z}^{2} & C_{z x}^{2}
\end{array}\right| \cdot\left|\begin{array}{lll}
x_{1} & y_{1} & 1 \\
x_{2} & y_{2} & 1 \\
x_{3} & y_{3} & 1
\end{array}\right|\right\}
\end{aligned}
$$

where

$$
d=\left|\begin{array}{ccc}
C_{y z}^{1} & C_{z x}^{1} & C_{x y}^{1} \\
C_{y z}^{2} & C_{z x}^{2} & C_{x y}^{2} \\
C_{y z}^{3} & C_{z x}^{3} & C_{x y}^{3}
\end{array}\right|
$$


For tetrahedra connected to the neighbors of the node $I_{I}$ we have:

$$
\begin{aligned}
& V^{\prime}=\left|\begin{array}{ccc}
x^{\prime}-x_{1} & y^{\prime}-y_{1} & z^{\prime}-z_{1} \\
x_{2}-x_{1} & y_{2}-y_{1} & z_{2}-z_{1} \\
x_{3}-x_{1} & y_{3}-y_{1} & z_{3}-z_{1}
\end{array}\right|=V+\Delta x \cdot\left|\begin{array}{ll}
y_{2} & z_{2} \\
y_{3} & z_{3}
\end{array}\right|+ \\
& +\Delta y \cdot\left|\begin{array}{ll}
z_{2} & x_{2} \\
z_{3} & x_{3}
\end{array}\right|+\Delta z \cdot\left|\begin{array}{ll}
x_{2} & y_{2} \\
x_{3} & y_{3}
\end{array}\right|,
\end{aligned}
$$

or

$V^{\prime}=V-\frac{1}{d} \alpha \Delta V_{3}\left\{\left|\begin{array}{ll}C_{z x}^{1} & C_{x y}^{1} \\ C_{z x}^{3} & C_{x y}^{3}\end{array}\right| \cdot\left|\begin{array}{ll}y_{2} & z_{2} \\ y_{3} & z_{3}\end{array}\right|+\left|\begin{array}{cc}C_{x}^{1} & C_{y z}^{1} \\ C_{x}^{3} & C_{y z}^{3}\end{array}\right| \cdot\left|\begin{array}{cc}z_{2} & x_{2} \\ z_{3} & x_{3}\end{array}\right|+\right.$

$\left.+\left|\begin{array}{cc}C_{y z}^{1} & C_{z x}^{1} \\ C_{y z}^{3} & C_{z x}^{3}\end{array}\right| \cdot\left|\begin{array}{ll}x_{2} & y_{2} \\ x_{3} & y_{3}\end{array}\right|\right\}-\frac{1}{d} \beta \Delta V_{2}\left\{\left|\begin{array}{cc}C_{z x}^{1} & C_{x y}^{1} \\ C_{z x}^{2} & C_{x y}^{2}\end{array}\right| \cdot\left|\begin{array}{ll}y_{2} & z_{2} \\ y_{3} & z_{3}\end{array}\right|+\right.$

$\left.+\left|\begin{array}{cc}C_{x y}^{1} & C_{y z}^{1} \\ C_{x y}^{2} & C_{y z}^{2}\end{array}\right| \cdot\left|\begin{array}{cc}z_{2} & x_{2} \\ z_{3} & x_{3}\end{array}\right|+\left|\begin{array}{cc}C_{y z}^{1} & C_{z x}^{1} \\ C_{y z}^{2} & C_{z x}^{2}\end{array}\right| \cdot\left|\begin{array}{cc}x_{2} & y_{2} \\ x_{3} & y_{3}\end{array}\right|\right\}$

Thus, volumes of all tetrahedra linearly depend on coefficients $\alpha$ and $\beta$ and, hence, either of the equations (5) and (6) constitute a plane relative to the coordinate system $(\alpha, \beta, V)$.

Similar equation systems can be also obtained for the nodes $I_{2}$ and $I_{3}$. As the values $\alpha_{i}$ and $\beta_{i}$ for each of the nodes $I_{1}, I_{2}$ and $I_{3}$ are related to one another then, to find their values, make the following operation. Denote as $\gamma_{i}$ the fraction of the $J_{k}$ cell volume which has to be recovered by displacement of the node $I_{i}$. Then equation systems (5) and (6) for the node $I_{1}$ can be re-written in the form

$$
V^{\prime}+\left(1-\gamma_{3}\right) \cdot A_{m}^{1}+\gamma_{1} \cdot B_{m}^{1}=V_{m}^{1}
$$

and for the node $I_{2}$

$$
V^{\prime}+\left(1-\gamma_{1}\right) \cdot A_{m}^{2}+\gamma_{2} \cdot B_{m}^{2}=V_{m}^{2}
$$

where $m=1, \ldots, 16$. At given values of $\gamma_{2}$ and $\gamma_{3}$ equation systems (7) and (8) determine a family of straight lines in tixe plane $\left(\gamma_{1}, V^{\prime}\right)$ (Fig.8). 


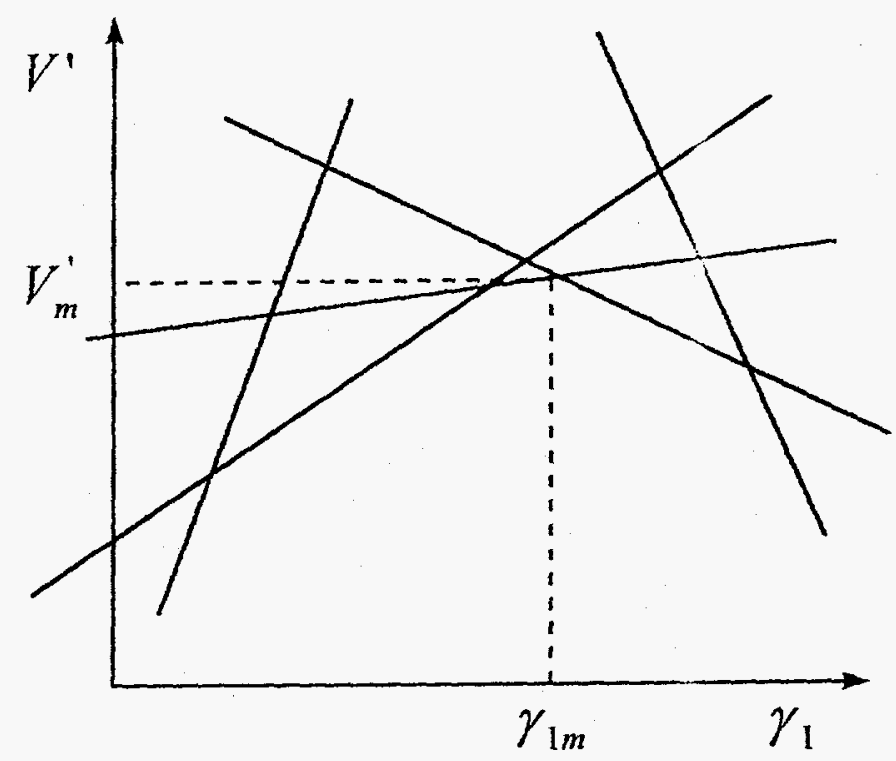

Family of straight lines in axes $\left(\gamma_{i}, V^{\prime}\right)$ determining the critical value of the parameter $V^{\prime}$

Fig 8.

When estimating the maximum of the minimum values $V$ ' we simultaneously estimate the value $\gamma_{1}$ at which absolute concavity values are minimal. Thus, one can arrange an iteraive process over all nodes $\boldsymbol{I}_{\boldsymbol{l}}$, $I_{2}$ and $I_{3}$ converging in $\gamma_{i}$ and allowing to compute their desired values, then the new coordinates of the nodes $I_{1}, I_{2}$ and $I_{3}$ are estimated uniquely. One may take the values $\gamma_{i}=1 / 2(i=1,2,3)$ as the initial data for the iterative process which corresponds to equal fractions of the volumes recovered by each of the nodes.

Thus, the problem of concavity correction is solved for one node. When there are concavities in several nodes, the correction begins with the node having the maximum concavity value modulo. The condition of concavity minimization satisfied at cell volume recovery leads to successive decrease in the maximum concavity value and eventually to correction of all concavities.

The problem of concavity correction in the internal node was considered above. When there are concavities in boundary nodes, the whole described procedure remains valid with the only difference that the equations of conservation of or variation in volumes of corresponding cells are replaced with the equations of the surface limiting the problem solution region.

The procedure of concavity correction with conservation of volumes can be used not only after local grid reconstructions, but also 
immediately for grid correction during gas-dynamical problem computation.

\section{CRITERIA FOR APPLICATION OF POLYHEDRAL 3D GRID LOCAL RECONSTRUCTIONS}

In the previous section we presented general description for local operations of polyhedral grid reconstruction. For automatic grid reconstruction during problem computation it is necessary to develop threshold criteria for execution of one or another grid reconstruction operation.

Basing on the experience of similar grid correction in two dimensions, for the parameters the grid state is estimated with we selected the following dimensionless values:

$\boldsymbol{R}_{I^{-}}$the ratio of the characteristic cell size to the length of the maximum cell diagonal;

$R_{2-}$ the ratio of the characteristic cell size to the value of this parameter averaged over the whole region;

$\boldsymbol{R}_{3^{-}}$the ratio of the cell volume to the average value of cells surrounding this cell;

$\boldsymbol{R}_{4^{-}}$the number of cell faces.

The characteristic cell size is estimated by the formula

$D=\sqrt{\frac{V}{d_{m}}}$ where $V$ - the cell volume, and $\boldsymbol{d}_{\boldsymbol{m}}$ - the maximum diagonal length. As the practice shows. such an approach reflects polyhedral cell features best.

Depending on the values of the estimating parameters $\boldsymbol{R}_{\boldsymbol{i}}$ the cut operation for a particular cell was executed if the following criteria were met:

1) $\left\{\begin{array}{l}R_{1}^{n}<R_{1}^{n-1} \\ R_{1}^{n}<0.25 \\ d_{m}^{n}>d_{m}^{n-1}\end{array}\right.$

where the superscript $\mathrm{n}$ is the number of the timestep, $d_{m}^{n}$ - the maximum cell diagonal at the $n$-th tim:estep. This condition may be interpreted as cut of cells tending to elongation. The number 0.25 corresponds to elongation of the initially unstrained Voronoi cell a little more than by a factor of two (for the Voronoi cell this parameter $\approx 0.6$, for a cube $\approx 0.44$ ). 
2) $\boldsymbol{R}_{3}>2$. This criterion prevents enlargement of individual cells possible from the paste operations.

3) $\boldsymbol{R}_{4}>30$. The sense of this criterion is evident, it counteracts appearance of cells with a great number of faces. Recall that the Voronoi body contains 14 faces, while a cell obtained from pasting two such cells along a hexagonal face has 20 faces.

Each cell was cut by a plane perpendicular to the straight line segment connecting centers of faces maximally apart from one another and halving the line.

A cell was pasted with one of its neighbors when the following criteria were met:

1) $\boldsymbol{R}_{2}<0.4$. Moreover, the number of cells covered by this criterion should not be higher than the number of cells cut by the criterion $\boldsymbol{R}_{1}$.

2) $\boldsymbol{R}_{3}<0.4$. The criterion serves to equalize grid cell sizes in a local vicinity, in particular, to remove small cells surrounded by larger neighbors.

3) $\boldsymbol{R}_{4}<5$. Removal of cells which are tetrahedra.

At cell paste of much importance is proper selection of a neighbor. The neighbor is selected by a set of the following criteria:

- the cell volume is minimal;

- the surface area of the face separating the cells to be pasted is maximal;

- the maximum diagonal of the cell obtained at the paste is minimal. By the parameter data set each neighbor candidate was granted a priority and the neighbor of maximum prio ity was selected.

As the methodical computation practice showed, these criteria for the polyhedral grid local reconstruction operations were quite sufficient to maintain the grid in a form correspondent to the Lagrangian computation requirements.

\section{MODEL COMPUTATIONS}

We used two model problems for testing the program. Setting up the first problem was kindly presented for us by Dr.D.Burton (LLNL). In this problem each grid node of the region which is originally a cube (Fig.9) is thrusted velocity estimated by the node coordinates and problem time. 


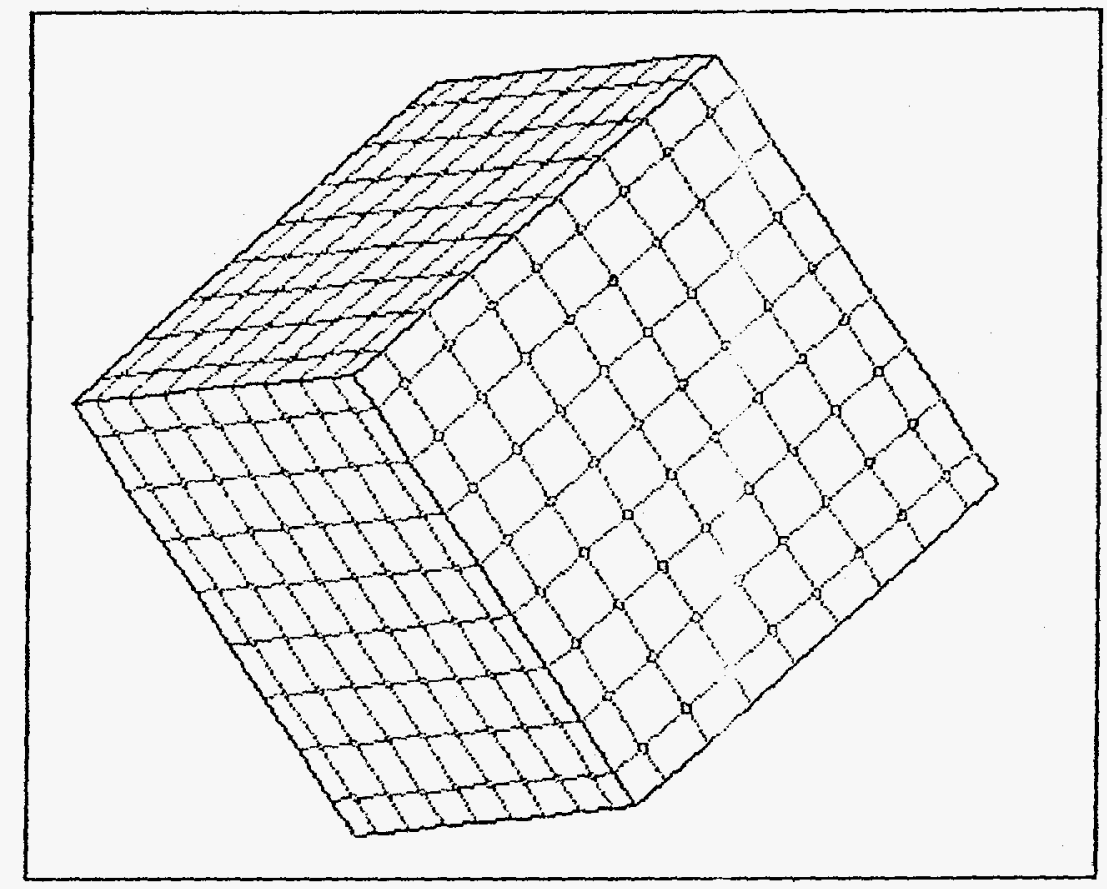

Projection of surface of a cube filled with polyhedral cells Fig 9.

The cube was filled with polyhed al cells with the Dirichlet method. The number of cells along the edge was 9 , the total number of cells was 1241 , of nodes 7016 .

The projections of the strained problem region at various times are given below (on the left without recons ructions, on the right with reconstructions).
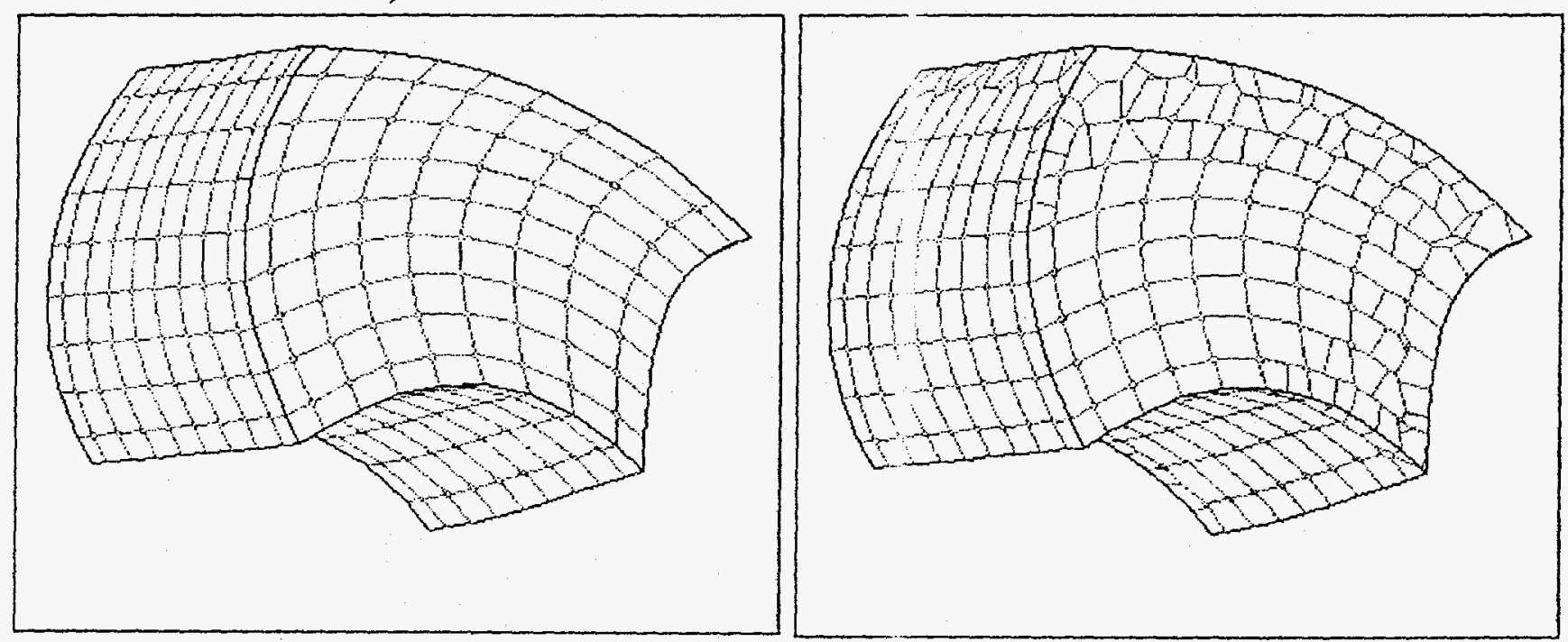

time $=0.1$ 

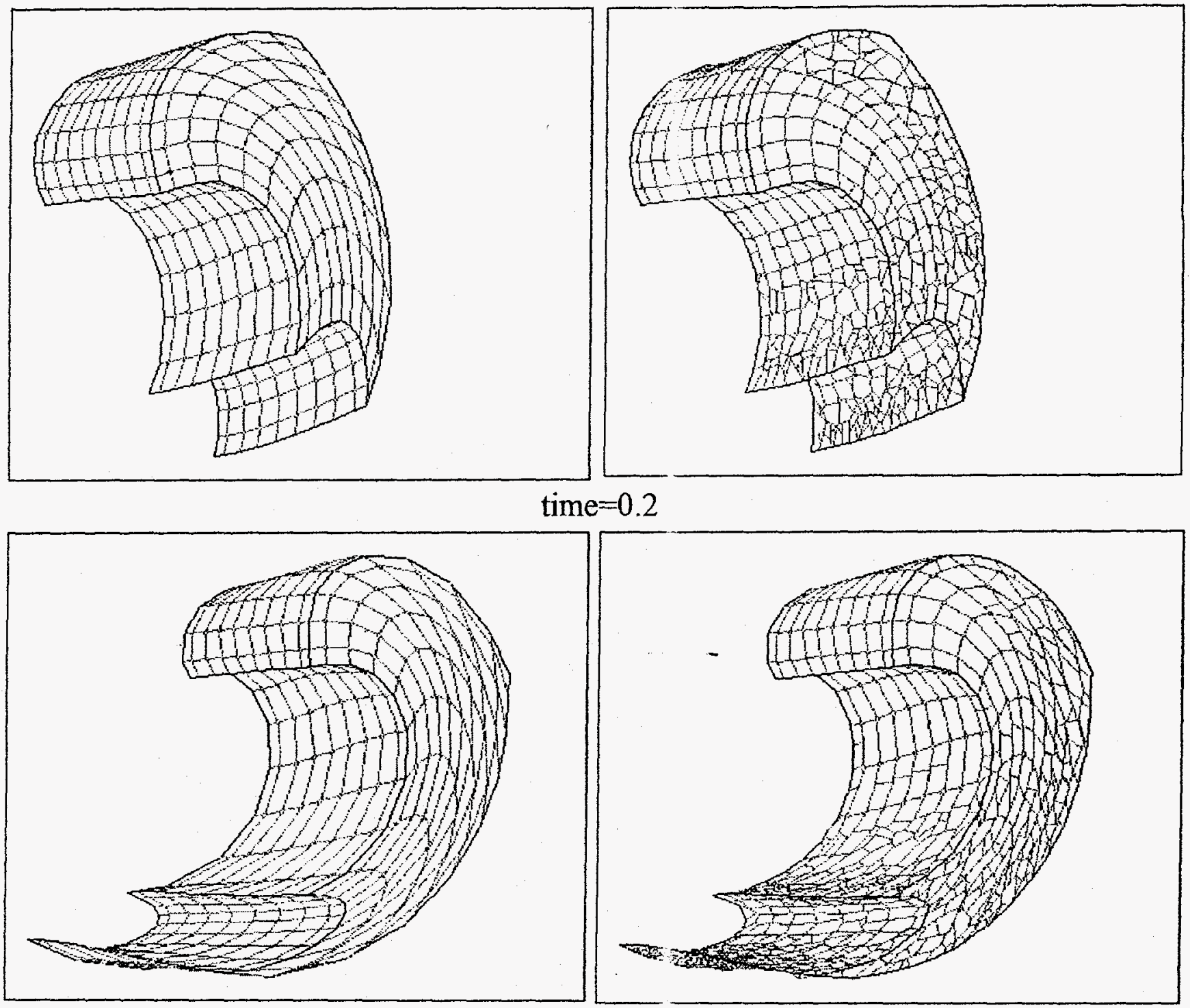

time $=0.3$
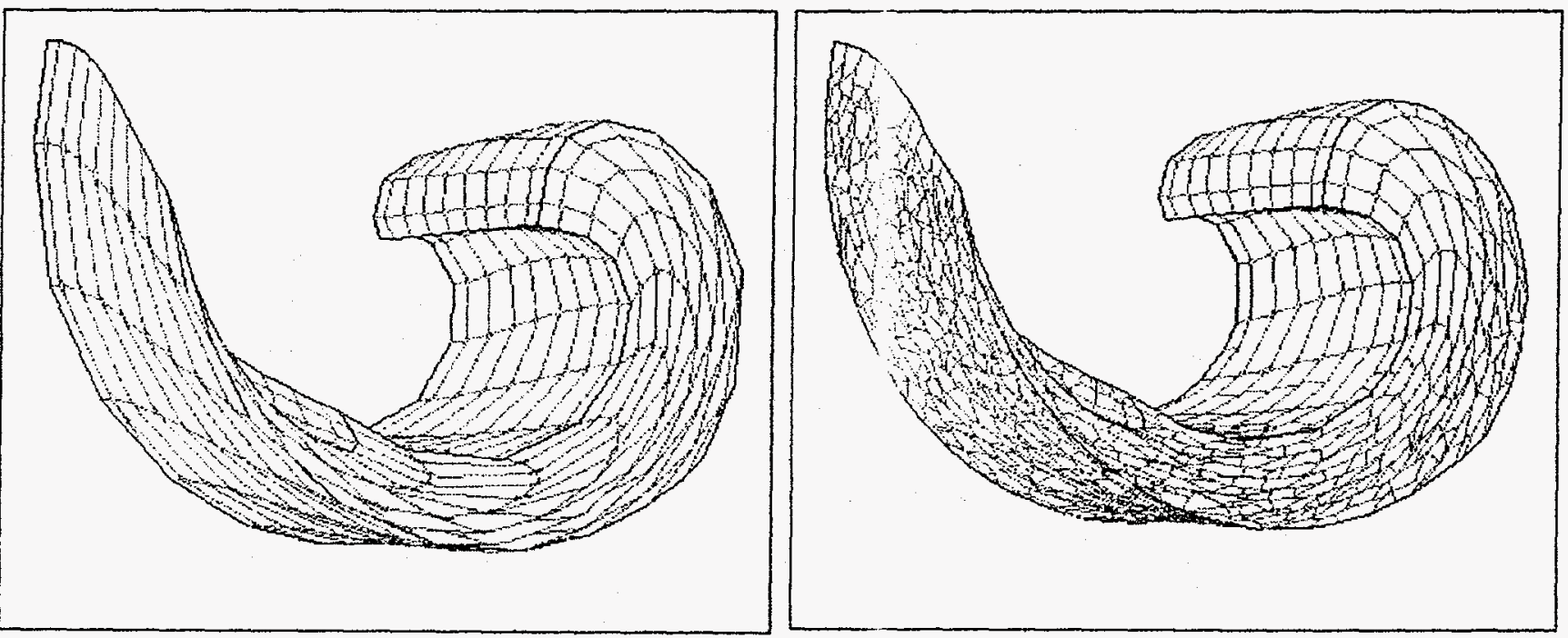

time $=0.4$ 
From the above figures it is seen that the unstrained region part cells were not subjected to reconstruction and, vice versa, a more active reconstruction took place in the region part where the cells were compressed and stretched during the problem computation.

The grid reconstruction program was called every 5 timesteps. First the computation was made at a constant step $\tau=10^{-4}$. From the time 0.2 a variable timestep was introduced which was estimated by the formula:

$$
\tau^{n+1}=\tau^{n} \frac{3 \cdot 10^{-3}}{\min _{i}\left(\frac{V_{i}^{n+1}-V_{i}^{n}}{V_{i}^{n}}\right)} \text { where } \mathrm{V}_{\mathrm{i}}^{\mathrm{n}} \text { - the volume of the } \mathrm{i} \text {-th cell at }
$$

the $\mathrm{n}$-th timestep.

Table 1 gives comparative characteristics for two versions of this problem computation.

Table 1

\begin{tabular}{|c|c|c|c|c|c|}
\hline & \multicolumn{2}{|c|}{$\begin{array}{c}\text { Computation without } \\
\text { reconstructions }\end{array}$} & \multicolumn{3}{c|}{$\begin{array}{c}\text { Computation with } \\
\text { reconstructions }\end{array}$} \\
\hline Time & $\begin{array}{c}\text { number } \\
\text { of cells }\end{array}$ & $\begin{array}{c}\text { maximum/ } \\
\text { minimum } \\
\text { characteristic } \\
\text { cell size }\end{array}$ & $\begin{array}{c}\text { number } \\
\text { of cells }\end{array}$ & $\begin{array}{c}\text { maximum/ } \\
\text { minimum } \\
\text { characteristic } \\
\text { cell size }\end{array}$ & $\begin{array}{c}\text { timestep } \\
\text { size } \tau\end{array}$ \\
\hline 0.0 & 1241 & 1241 & & $1 \cdot 10^{-4}$ \\
\hline
\end{tabular}




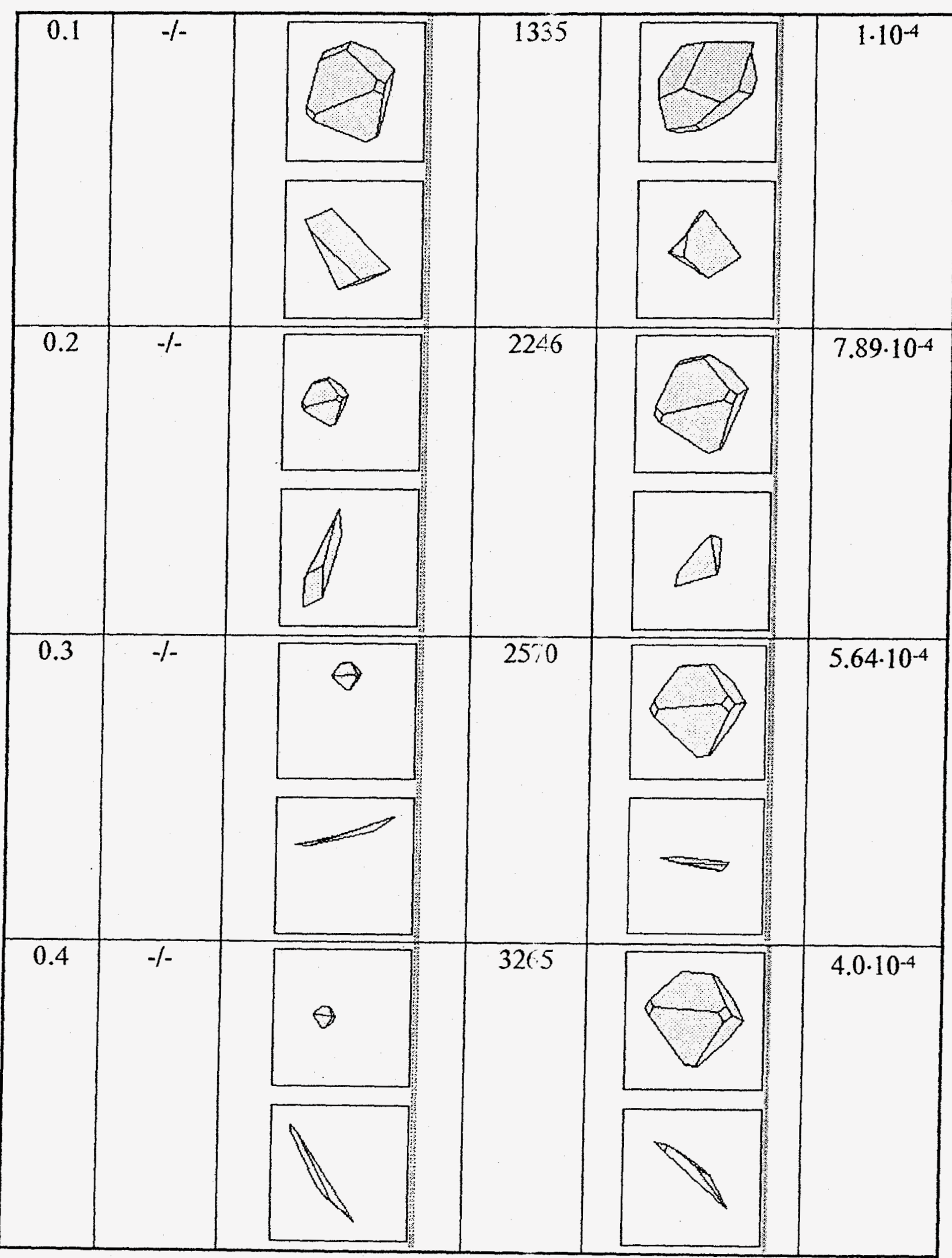


Activity of the grid reconstruction program operation increases as the region strain grows. The maximum quantity of cut cells at one program call was $\sim 40$, of pasted cells $\sim 30$. From the above results it is seen that in the computation with grid reconstructions the cells are more compact than in the computation without reconstructions. It should be noted that in the problem the flow character itself does not lead to degeneration of cells to self-intersecting cells in the computation without grid reconstructions. At worst, they become stretched and non-convex.

We made one more model computation where the polyhedral grid reconstruction program was used more intensely.

Like in the first computation, strain of the region which originally was a cube depended on a given velocity field. Velocity in each grid node was determined by a set of relations:

$$
\begin{aligned}
& U_{x}=0, \\
& U_{y}=U_{r} \operatorname{Cos}(\varphi)+U_{\varphi} \operatorname{Sin}(\varphi), \\
& U_{z}=U_{r} \operatorname{Sin}(\varphi)-U_{\varphi} \operatorname{Cos}(\varphi),
\end{aligned}
$$

where

$$
\begin{aligned}
& U_{r}=\frac{4 \cdot\left(\left|x_{i}-x_{c}\right|-\frac{1}{2} a\right)^{2} \cdot r_{i}}{a^{2}(t-1)}, \\
& U_{\varphi}=\frac{4 \pi r_{i}^{2}\left(x_{i}-x_{c}\right)\left|x_{i}-x_{c}\right|}{a^{3}(t-1)}, \\
& r_{i}=\sqrt{\left(y_{i}-y_{c}\right)^{2}+\left(z_{i}-z_{c}\right)^{2}}, \\
& \operatorname{Sin}(\varphi)=\frac{\left(y_{i}-y_{c}\right)}{r_{i}}, \operatorname{Cos}(\varphi)=\frac{\left(z_{i}-z_{c}\right)}{r_{i}} .
\end{aligned}
$$

Here $\boldsymbol{a}$ - the cube edge size, $\mathrm{x}_{c}, \mathrm{y}_{\mathrm{c}}, \mathrm{z}_{\mathrm{c}}$ - cube center coordinates, $t$ problem time. The cube was filled with polyhedral cells with the Dirichlet method. The number of cells along the edge was equal to 5 , the total number of the cells to 189 .

The projections of the strained probiem region at various times are given below (on the left without reconstructions, on the right with reconstructions). 
$9^{\circ} 0=1$

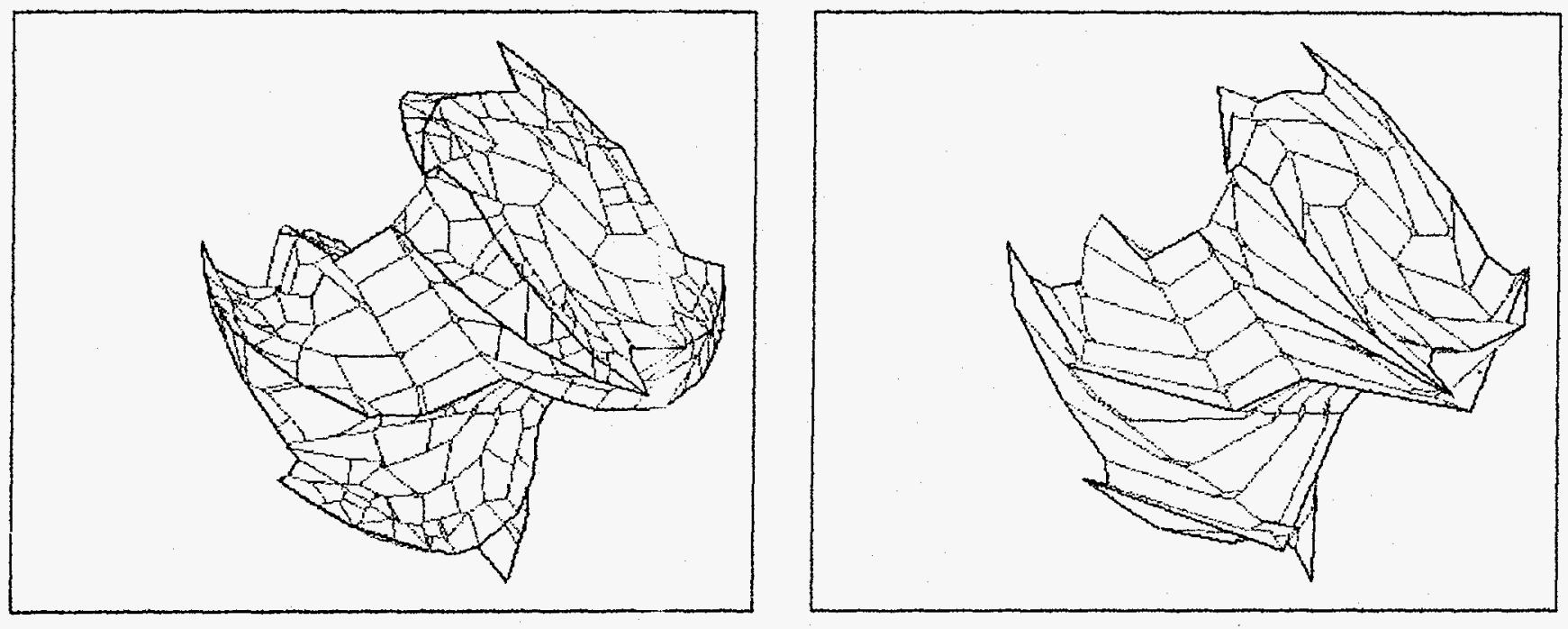

$\rightarrow 0 \Rightarrow$
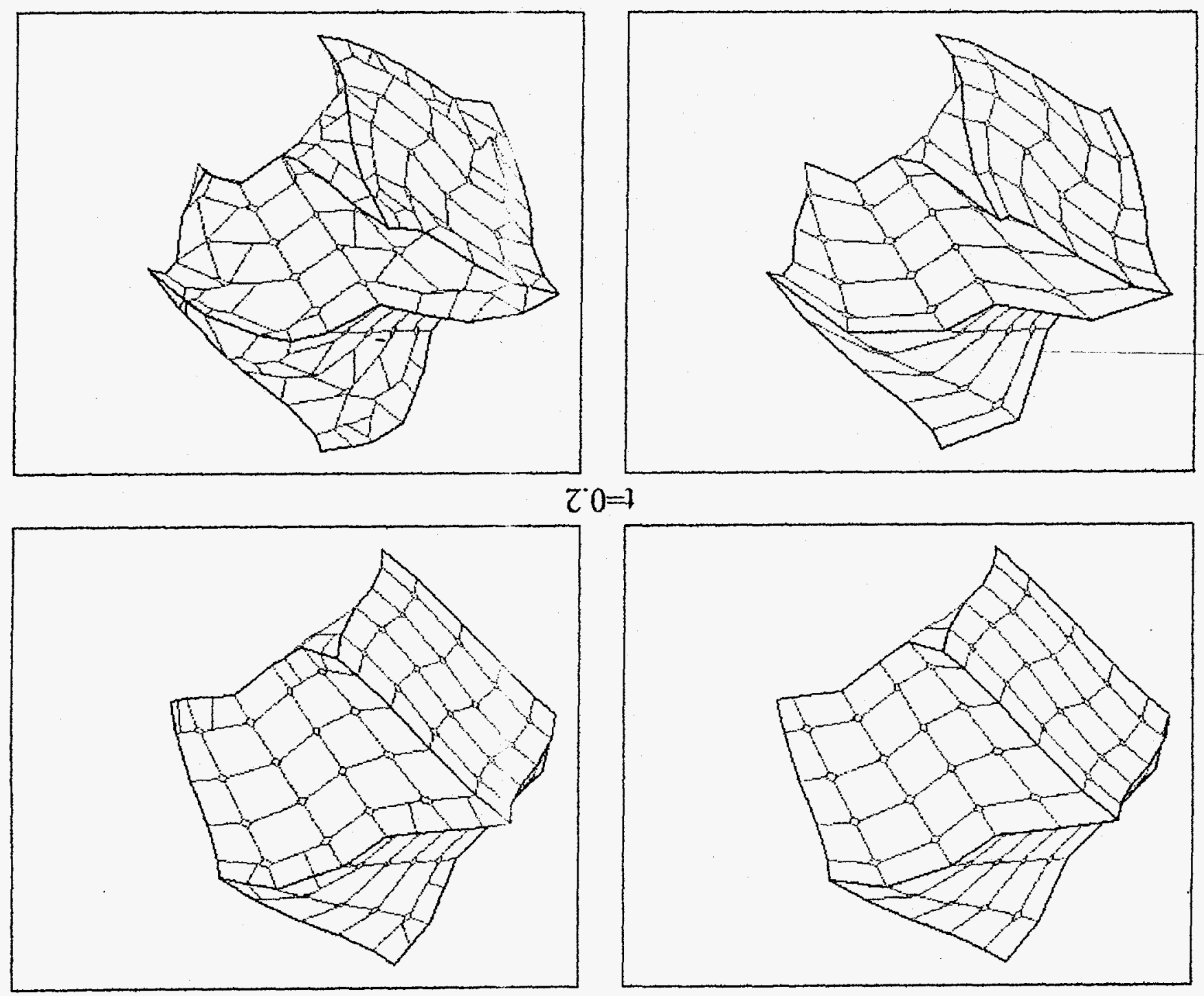

$20 \Rightarrow$ 

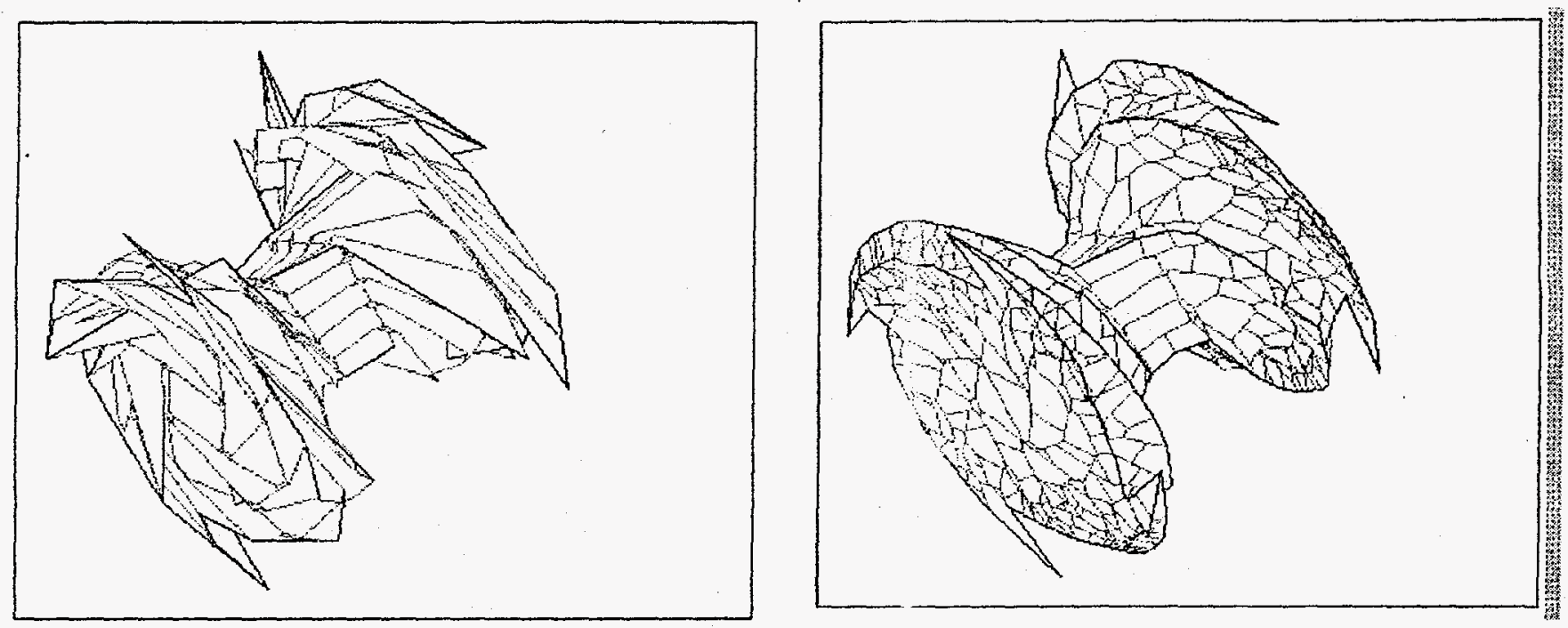

$t=0.8$

In contrast to the previous problem, in this problem in the computation without reconstructions by time $t=0.6$ cells of negative volume appear. This, of course, does not take place in the computation with reconstructions. The timestep size in the computation with reconstructions was estimated by the same formula as in the first problem. In the computation without reconstructions the timestep was limited from below with the constant $10^{-3}$. Table 2 gives comparative characteristics for two versions of this problem computation.

Table 2

\begin{tabular}{|c|c|c|c|c|c|}
\hline & \multicolumn{2}{|c|}{$\begin{array}{l}\text { Computation without } \\
\text { reconstructions }\end{array}$} & \multicolumn{3}{|c|}{$\begin{array}{l}\text { Computation with } \\
\text { reconstructions }\end{array}$} \\
\hline Time & $\begin{array}{l}\text { number } \\
\text { of cells }\end{array}$ & $\begin{array}{c}\text { maximum/ } \\
\text { minimum } \\
\text { characteristic } \\
\text { cell size }\end{array}$ & $\begin{array}{l}\text { number } \\
\text { of cells }\end{array}$ & $\begin{array}{l}\text { maximum/ } \\
\text { minimum } \\
\text { characteristic } \\
\text { cell size }\end{array}$ & $\begin{array}{l}\text { timestep } \\
\text { size } \tau\end{array}$ \\
\hline 0.0 & 189 & & 189 & & $1.73 \cdot 10^{-3}$ \\
\hline
\end{tabular}




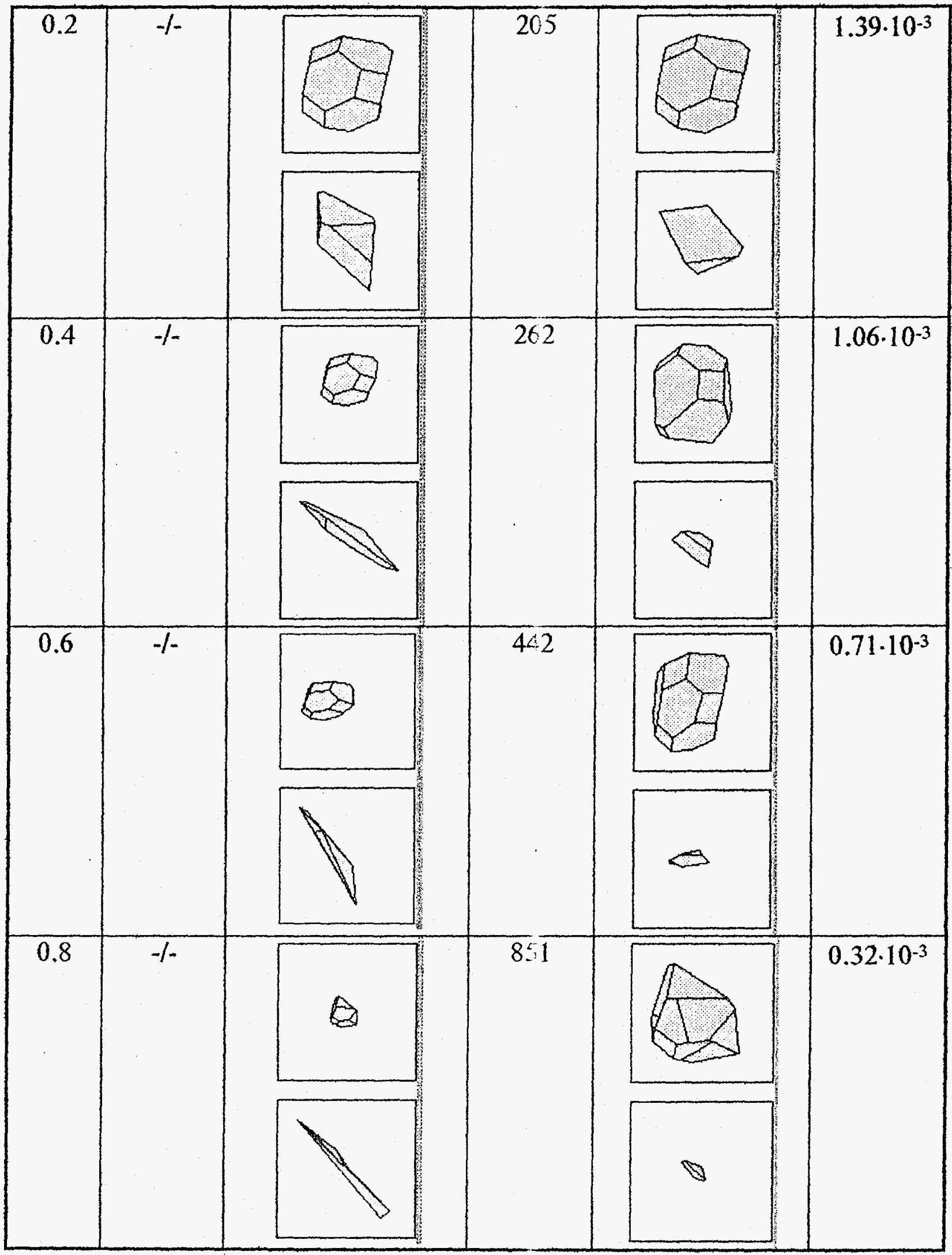


The local reconstruction program is written in FORTRAN-77. The number of the FORTRAN program operators is 6800 .

The first model computation was used to debug the main program nodes and eliminate defects, therefore, here we do not present any calendar dates for this computation. The second computation was control and run without any aborts. The problem was computed up to time 0.8. 950 timesteps were therewith executed. The calendar time of the computation was 3 hours of PC/AT-586 $(90 \mathrm{MHz})$ computer.

\section{CONCLUSION}

The algorithm is developed and the program is written for regridding during a gas-dynamical computation consisting in paste and cut of particular cells. Threshold criteria for local reconstruction during the gas-dynamical computation are developed. The program was used to conduct two computations of model problems demonstrating polyhedral grid local reconstruction algorithm and program performance.

We believe that the criteria of demand for reconstruction and the algorithms of its implementation are not complete. Most probably, they will be improved and modified during program operation. We hope that performance of the work under this contract will be a key for proceeding with further collaboration of our laboratories in the area of development of efficient programs designed for computing problems of gas dynamics with involvement of other physical processes.

\section{REFERENCES}

1. Fedorov E.S. Regular plane and space division. Moscow, Nauka Publishers, 1979.

2. Vainshtein B.K. Modern crystallogaphy. V.1, Moscow, Nauka Publishers, 1979.

3. Delaunay B.N. Theory of numbers Petersburg school. MoscowLeningrad, USSR Academy of Sciences Publishing House, 1947.

4. Sofronov I.D., Rasskazova V.V., Nesterenko L.V., " The Use of Nonregular Nets for Solving Two - Dimensional Nonstationary Problems in Gas Dynamics", Numerical Methods in Fluid Dynamics Edited by N.N. Yanenko and Yu.I. Sh $\circ$ kin 1985. 